\title{
Simple Shearing Flows in Polyisobutylene Solutions
}

\author{
L. J. Zapas and J. C. Phillips \\ Institute for Basic Standards, National Bureau of Standards, \\ Washington, D.C. 20234
}

(July 2, 1970)

\begin{abstract}
Measurements of shear stress for various shearing flow histories at $25{ }^{\circ} \mathrm{C}$ are correlated through the BKZ elastic fluid theory. The data are on 10 percent PIB solution in cetane. The histories include single step stress relaxation, suddenly applied steady shear, steady shearing flow, stress relaxation after steady shear.
\end{abstract}

Key words: BKZ fluid; nonlinear behavior; polyisobutylene; stress relaxation; viscosity.

\section{Introduction}

In this paper we present the results of a series of experiments on a single sample of polyisobutylene solution subjected to various histories of simple shear. The work was done as a part of a larger program to study the appropriateness of a fairly general constitutive equation, the Bernstein, Kearsley, Zapas (BKZ) elastic fluid $[1],{ }^{1}$ in describing a wide range of nonlinear viscoelastic behavior for a range of materials. This larger purpose influenced both the choice of test material and the choice of experiments. In the first place, the studies were confined to a single sample so as to provide a test of the correlations between experiments predicted by the assumed constitutive equation. The sample was chosen to show essentially complete relaxation within the time scale of the experiment, since earlier studies [2] had been made on materials with longer relaxation times. The deformations are various histories of simple shear, because they are experimentally convenient deformations and because they are convenient to analyze in terms of the assumed constitutive equation.

Some measurements of normal stresses were made on this sample, but we encountered serious discrepancies when we attempted to correlate them with the shear data. This trouble has been traced to the recently discovered "pressure hole error" [3] and the difficulty resolved, but we have postponed publication of the normal stress data to avoid further delay in the presentation of the shear stress measurements.

In the course of these studies some measurement errors, customarily ignored, were found to be surprisingly large and some correction methods were worked out and are presented here. A specialized form of the BKZ elastic fluid was used in this paper to

'Figures in brackets indicate the literature references at the end of this paper. correlate our experiments with very good success. Although this special form is neither unique nor exact, it has been chosen to be consistent with a wide range of phenomena beyond that described in this paper, and should be of considerable practical use.

\section{General Considerations}

The class of motions which we shall consider will be limited to simple shearing histories.

In the incompressible isothermal form of the BKZ theory $[1,4]$ the value of the shearing stress at time $t$ is given by the following relation

$\sigma(t)=$

$$
-\int_{-\infty}^{t} W_{*}(\gamma(t)-\gamma(\tau), t-\tau)(\gamma(t)-\gamma(\tau)) d \tau
$$

where $\gamma(\tau)$ is the amount of shear at time $\tau$ and $W_{*}$ is a function of strain and time. ${ }^{2}$ If $W_{*}$ is known, the shearing stress $\sigma(t)$ for any simple shearing flow can be calculated through eq (2.1). Of course, the form of $W_{*}$ is not specified by the theory and in general the function $W_{*}$ must be determined through experiments.

A good idea of the function $W_{*}$ can be obtained from examining a single step stress relaxation history in simple shear. In such a deformation the shearing history is given by:

$$
\begin{array}{ll}
\gamma(\tau)=0 & \text { for } \tau<0 \\
\gamma(\tau)=\gamma=\text { constant } & \text { for } \tau>0 .
\end{array}
$$

By substituting $\xi=t-\tau$ and (2.2) in (2.1), we get

$$
\frac{\sigma(t)}{\gamma}=-\int_{t}^{\infty} W_{*}(\gamma, \xi) d \xi=W(\gamma, t)
$$

${ }^{2}$ Equation (2.1) is equivalent to eq (3.11) of Bernstein [4] except for a change of notation. $W_{*}$ of this equation is equal to $-2\left(U_{l_{1}}+U_{l_{2}}\right)$ of Bernstein, and our $\sigma(t)$ is Bernstein's $\sigma_{12}$ 
where

$$
W_{*}(\gamma, t)=\frac{\partial W(\gamma, t)}{\partial t}
$$

and

$$
W(\gamma, \infty)=0
$$

In this paper we shall refer to $W$ as the relaxation function. From (2.3) it follows that $W$ or $W_{*}$ may be obtained from single step stress relaxation measurements in shear at different strains and from this the stress for any simple shearing flow history may be predicted. This approach is similar to the one used for simple extension histories [2].

Bernstein [5] has shown that the function

$$
\mathscr{H}(\gamma, t)=W(\gamma, t)\left(1+\frac{\partial \ln W(\gamma, t)}{\partial \ln \gamma}\right)
$$

may also be obtained from experiments of suddenly applied steady shear. Thus, in principle, we should be able to obtain $W(\gamma, t)$ from a series of experiments, and then proceed to check the BKZ theory by comparing measured and predicted stress for simple shearing histories.

Of course, one can never realize experimentally these shearing histories exactly as assumed. From some of the experiments which approximated suddenly applied constant rate of shear history, discussed in section 6 , we were able to get a rough approximation of the relaxation function. Applying corrections which will be discussed later using an iterative scheme, we recalculated a function $W$ which is consistent with all our experiments which include measurements of viscosity as a function of the rate of shear, stress relaxation after shear (for different rates of shear), measurement of stress as a function of time for suddenly applied steady shear, and single step stress relaxation experiments. These results, we felt, justified the use of an expression for $W(\gamma, t)$ which can describe all our experiments and which is a special form of a more detailed expression consistent with the behavior of other materials and other deformations, viz:

$$
W(\gamma, t)=G(t)\left\{\frac{1}{1+\frac{1}{3} \gamma^{2}}+\frac{0.145|\gamma|}{\left(1+0.06 \gamma^{2}\right)^{5 / 2}}\right\}
$$

where $G(t)$ is the shear stress relaxation modulus. ${ }^{3}$ It is evident that there are many other forms which are consistent with our experimental data. Our motivation for choosing form (2.6) is its relative simplicity and its consistency with a strain potential function [1]. Furthermore, form (2.6) is useful in process control engineering since an immediate translation from the linear to the nonlinear behavior can be made as a

\footnotetext{
${ }^{3}$ Notice that $G(t)$, the shear relaxation modulus, is the limit of relaxation function $W(\gamma, t)$ as $\gamma$ goes to zero.
}

function of temperature, molecular weight, and concentration.

\section{Experimental}

The data reported in this paper were obtained on a 10-percent solution of polyisobutylene (vistanex $\mathrm{L}-100$, Enjay Chemical Co. $)^{4}$ in cetane. A Weissenberg Rheogoniometer was used to shear the sample between a flat plate $(7.5 \mathrm{~cm}$ diameter) and a cone such that the angle of the gap was 0.0268 radians. The cone was at the bottom and was connected to the driving shaft. The plate was at the top and was connected to a torsion bar which was used to measure the torque. For most of the experiments a torsion bar of $1 / 8$-in diameter was used. The stress-time measurements and the dynamic response were recorded on an oscillograph.

The chamber enclosing the cone-plate assembly was kept at a temperature of $25.0^{\circ} \mathrm{C} \pm 0.1^{\circ} \mathrm{C}$. Ambient temperature was controlled at $25.0^{\circ} \mathrm{C} \pm 0.5^{\circ} \mathrm{C}$. An ambient temperature 3 degrees below the chamber temperature caused a noticeable change in the curve of viscosity versus rate of shear at high rates of shear. The zero shear viscosity at this temperature varies about 5 percent per degree.

Periodically, degradation checks were made on samples of 10-percent PIB by taking viscosity versus rate of shear data. No changes were observed over a 2 year period.

\section{Behavior at Small Deformations}

It is clear from eq $(2.6)$ that the shear relaxation modulus, $G(t)$, plays an important role in our curve fitting scheme. Since stress relaxation measurements cannot be carried out using deformations small enough to yield an infinitesimal modulus, this is ordinarily obtained by some sort of extrapolation procedure. An alternate method, adopted here, is to calculate a curve from measured values of the dynamic modulus, $G^{\prime}(\omega)$ [6]. We employed the conversion scheme given by Marvin [7],

$$
\begin{aligned}
& G(t)=\left.G^{\prime}(\omega)\right|_{1 / \omega=t} \\
& \quad-\int_{-\infty}^{\infty} H(\tau)\left(\frac{\tau^{2}}{t^{2}+\tau^{2}}-e^{-t / \tau}\right) d \ln \tau,
\end{aligned}
$$

using an approximation technique similar to the familiar derivative schemes [6] for the relaxation spectrum, $H(\tau)$. Even though we must depend on extrapolation for values of $H(\tau)$ at small $\tau$, the effect on $G(t)$ is slight.

Figure 1 shows the dynamic data and figure 2 the derived values of $G(t)$. The dotted portion of figure 2 represents values extrapolated to times smaller than

${ }^{4}$ Certain commercial equipment, instruments, or materials are identified in this paper in order to adequately specify the experimental procedure. In no case does such identification imply recommendation or endorsement by the National Bureau of Standards, nor does it imply that the material or equipment identified is necessarily the best available for the purpose. 
$\frac{\sigma(t)}{\gamma} / W(\gamma, t)$ goes to unity at high values of $t$.

For values of $\gamma$ such that

$$
\left|W_{*}(\gamma, t)\right| \geqslant\left|\alpha W_{*}(\alpha \gamma, t)\right|, \quad 0 \leqslant \alpha \leqslant 1
$$

a condition satisfied in the stress relaxation data reported here, it can be shown [8] that $\sigma\left(t+t_{1}\right) / \gamma$ for $t \geqslant t_{1}$ is a lower bound of $W(\gamma, t) .^{5}$

An example of the two bounds and the function $W(\gamma, t)$ is shown in figure 3 for $\gamma=0.2$ and $t_{1}=0.03 \mathrm{~s}$. The values of $W(\gamma, t)$ are the values that we used to correlate our different experiments. We see that even for values of $t=10 t_{1}$ the upper bound is high. The average of the two bounds gives agreement to within 1 percent. In the example in figure 3 , a very good approximation of $W(\gamma, t)$ can be obtained by averaging the time of the two bounds, viz,

$$
\frac{\sigma\left(t+\frac{1}{2} t_{1}\right)}{\gamma} \simeq W(\gamma, t)
$$

In our experiments the strains were induced through a shaft of the rheogoniometer normally used for sinusoidal deformation histories. The motion of the cone was monitored with a transducer. The deformations were obtained by using a springloaded lever arm to drive the shaft through part of a cycle. This arrangement allowed us to obtain strains up to $\gamma=1.8$. By using only a part of the sinusoidal deformation, we could obtain a motion of the cone very close to a ramp function. The time required to reach the maximum strain was of the order of 0.01 to $0.05 \mathrm{~s}$.

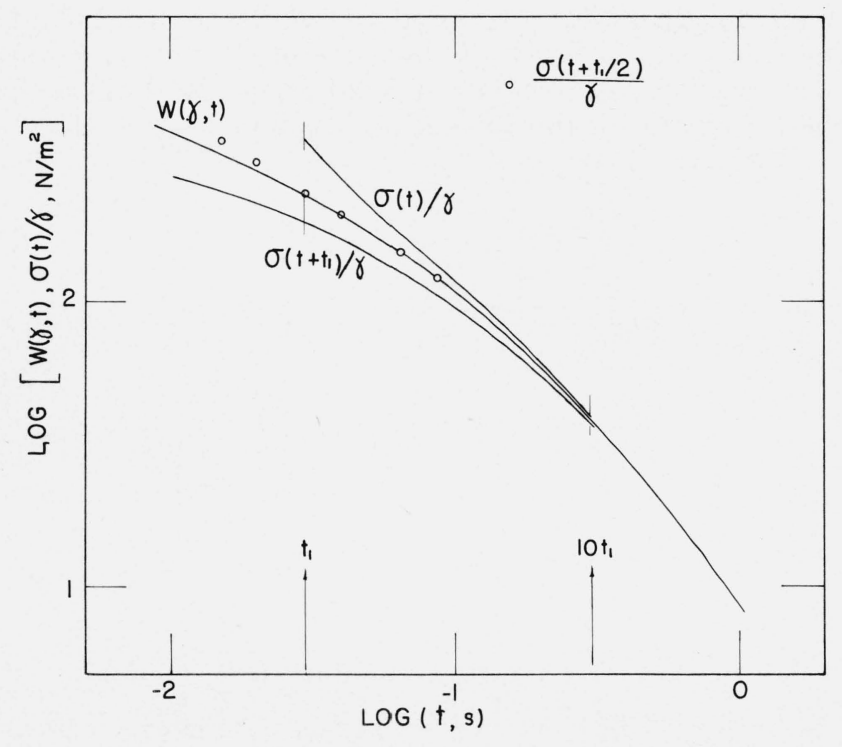

Figure 3: Bounds of $\mathrm{W}(\gamma, \mathrm{t})$ for $\gamma=0.2$ and $\mathrm{t}_{1}=0.03 \mathrm{~s}$.

${ }^{5}$ This is equivalent to taking the relaxation from the instant of reaching the final shear value.
Unfortunately, in our experimental system there is another complication due to the motion of the upper platen. The true strain at any time $t$ is more nearly $g(t)-\frac{\sigma(t)}{K}$ where $K$ is a constant depending on the stiffness of the torsion bar and the geometry of the cone and plate, and $g(t)$ is the nominal strain at $t$ calculated from the motion of the cone. With a stiffer torsion bar the error due to the motion of the upper plate would be smaller but small stresses could not be measured with enough precision. If the motion of the cone is monitored, one can calculate the error due to the motion of the plate. In our measurements the error in the stress at the early times was 6 percent.

Figure 4 shows a plot of $W(\gamma, t)$ versus $\gamma$ in which both corrections were made. The isochrones at the early times are in very good agreement with eq (2.6) which corresponds with the lines. To the extent that the isochrones are parallel in this type of a plot, i.e., superposition occurs by a vertical translation, one can justify the representation of $W(\gamma, t)$ as a product of the stress relaxation modulus and a function of shear, $\gamma$, i.e., eq (2.6).

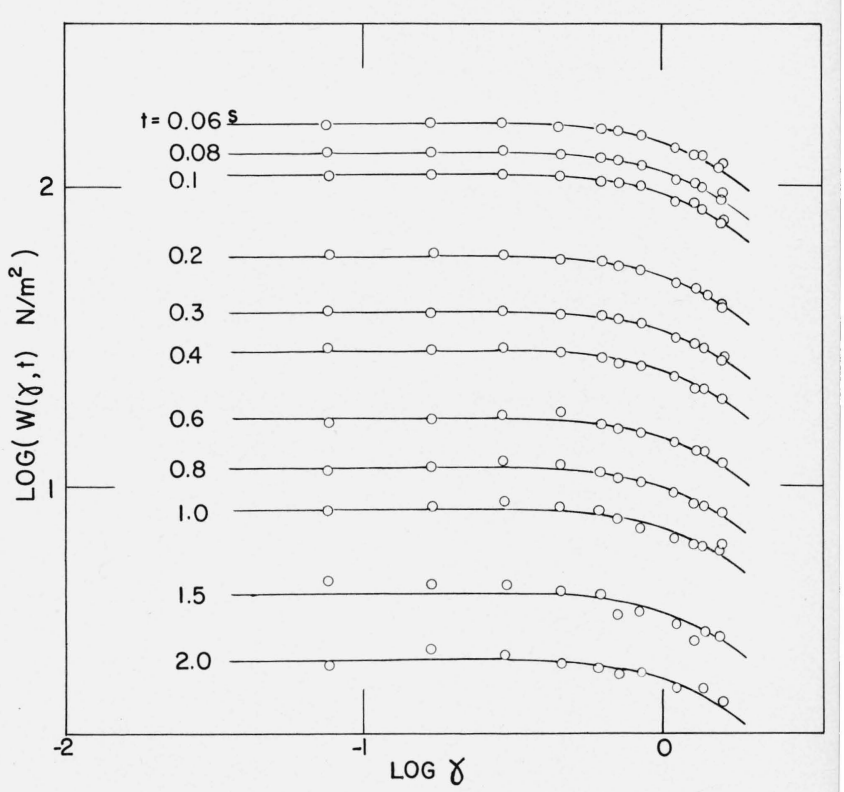

FigURE 4: Isochrones of $\mathrm{W}(\gamma, \mathrm{t})$ versus $\gamma$.

The lines indicate the calculated behavior from eq (2.6).

\section{Suddenly Applied Constant Rate of Shear}

With our experimental arrangement we found that we could not obtain an instantaneous constant rate of shear. For that reason we monitored and measured the motion of the cone with a transducer during the early part of the shearing. The monitoring arrangement prevented the use of the temperature control chamber. Therefore, the measurements were done at room tem- 


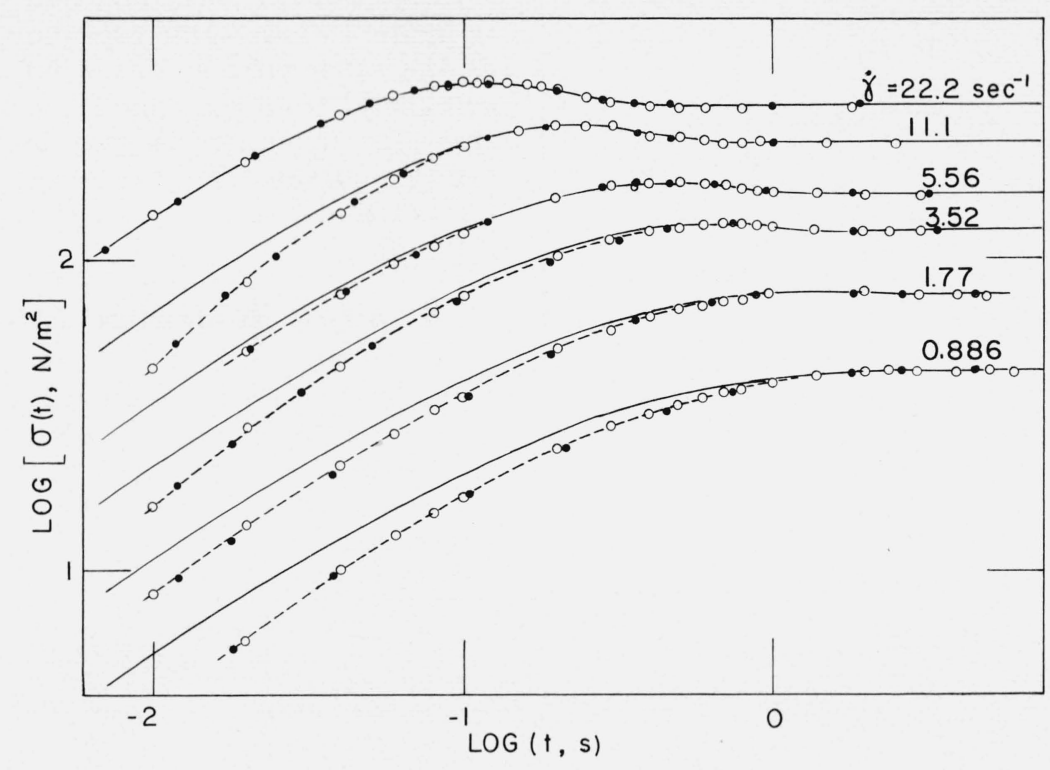

FIGURE 5: The solid lines represent the stress calculated for a suddenly applied rate of shear for the rates of shear indicated.

The open circles are experimental data, and the solid circles are calculated values using the true history of the motion.

perature. Since it took about $20 \mathrm{~s}$ to complete a run, the temperature variation was no more than $\pm 0.5^{\circ} \mathrm{C}$ at $25^{\circ} \mathrm{C}$.

For these runs, due to the limitations of the transducer, we could monitor strains only up to $\gamma=0.4$. On repeating an experiment, different strain histories were invariably obtained (over the early part of the time scale); this was probably caused by differing positions of the gear teeth. So, from each series of repetitive runs, we selected the one with the least deviation from a constant rate of shear.

Using eq (2.1) with the history

$$
\begin{aligned}
& \gamma(\tau)=0, \quad \tau<0 \\
& \gamma(\tau)=\dot{\gamma} \tau, \tau \geqslant 0 ; \quad \dot{\gamma}=\mathrm{constant}
\end{aligned}
$$

we obtain ${ }^{6}$

$$
\sigma(t)=\dot{\gamma} \int_{0}^{t} W(\dot{\gamma} \xi, \xi)\left(1+\frac{\partial \ln W(\dot{\gamma} \xi, \xi)}{\partial \ln \gamma}\right) d \xi
$$

where $\gamma=\dot{\gamma} \xi$ and $\dot{\gamma}$ is the rate of shear. In figure 5 we show the data as obtained. The lines represent the prediction of (6.2) and (2.6) which assume a suddenly applied steady shear (for the rates of shear indicated), and the black solid circles are values calculated from (2.1) and (2.6) using the true history of the motion. The agreement is very good. The inertia of the upper platen and assembly was not corrected for. We estimated for $\dot{\gamma}=22.2 \mathrm{~s}^{-1}$ that the inertial effect caused an error of

\footnotetext{
${ }^{6}$ Throughout the paper we shall use the notation $\frac{\partial \ln W}{\partial \ln \gamma}$ to represent the derivative of ln $W$ with respect to the derivative of the logarithm of the first argument.
}

less than 2 percent. Figure 5 shows at high rates of shear an overshoot of the stress $\sigma(t)$ as it approaches its limiting value.

From eq (6.2) the maximum value of the stress should occur when

$$
\frac{\partial \ln W(\gamma, t)}{\partial \ln \gamma}=-1
$$

Our empirical form of $W(\gamma, t)$, eq (2.6), satisfies this condition at $\gamma=2.44$.

In table 2 we show the strains at which the maximum of the stress occurs from other data. ${ }^{7}$ Middleman's data [9] were estimated from the published graphs. The data of the more concentrated polyisobutylene solutions were obtained in our laboratory. The zero shear viscosities at $25{ }^{\circ} \mathrm{C}$ were 4460 poise for the 15.1 percent concentration and 17,760 poise for the 19.3percent concentration. Assuming the principle of reduced variables with respect to concentration to be valid [10], we see that the rates of shear reported in table 2 for the higher concentrations will be 8 times as high when reduced to 10-percent concentration, for which the zero shear viscosity is 540 poise. $^{8}$

The position of this overshoot can be used to study the error caused by the motion of the upper platen. It is easy to show this error by experiments with torsion bars of different diameters. In figure 6 we show the data obtained from a 15.1 percent solution at a nominal

\footnotetext{
${ }^{7}$ If eq (2.6) is correct for all concentrations, the maximum of the stress must occur a the same value of $\gamma$

${ }^{8}$ The reduced rate of shear $\dot{\gamma}_{R}$ is given as

$$
\dot{\gamma}_{R}=\dot{\gamma} \frac{\eta_{1}}{\eta_{2}}\left(\frac{c_{1}}{c_{2}}\right)^{2}
$$
}

where $\eta_{i}$ is the zero shear viscosity at concentration $c_{1}$. 


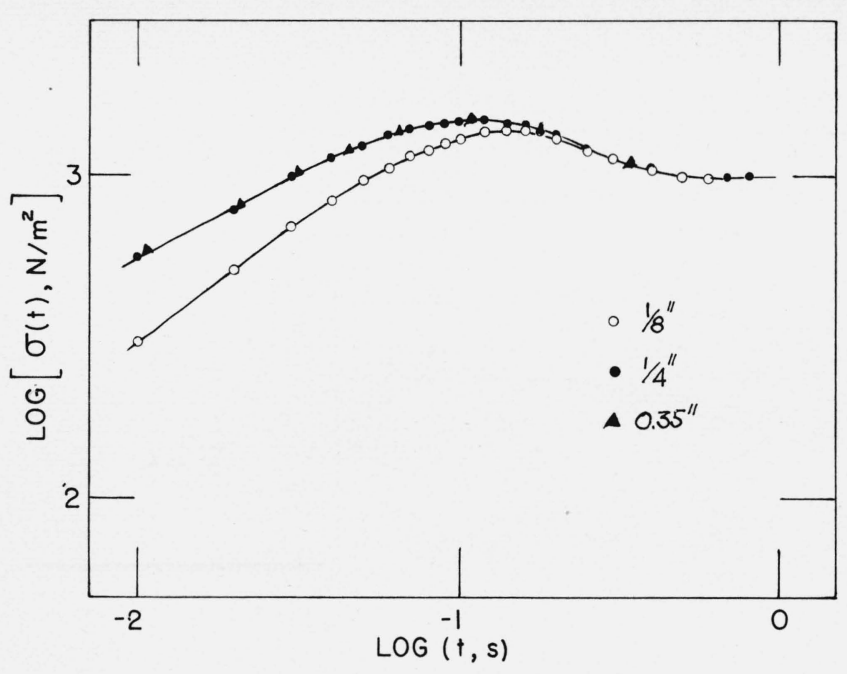

Figure 6: Stress versus time, using different torsion bars for the same nominal rate of shear of $22.2 \mathrm{~s}^{-1}$ applied at $\mathrm{t}=0$, for a 15.1percent solution of $P I B$ in cetane.

rate of shear of $22.2 \mathrm{~s}^{-1}$. The open circles show the data obtained with a 0.125 -in torsion bar. The solid circles are data obtained with a 0.25 -in bar and the black triangles, with a 0.35 -in bar. The histories of the motion of the cone for all the runs were almost identical, so that a direct comparison can be made of the effect of the motion of the upper platen. The maximum occurs at $t=0.11 \mathrm{~s}$ for the 0.25 -in and 0.35 -in bars and at $t=0.16 \mathrm{~s}$ for the 0.125 -in bar.

TABLE 2. Values of $\gamma$ at which the maximum of the shearing stress occurs for the designated rates of shear

\begin{tabular}{c|c|c|c}
\hline \hline$\dot{\gamma}$ & $\begin{array}{c}15.1 \text { percent } \\
\text { Vistanex L-100 } \\
\text { (in cetane) } \\
\text { at } 25{ }^{\circ} \mathrm{C}\end{array}$ & $\begin{array}{c}19.3 \text { percent } \\
\text { Vistanex L-100 } \\
\text { (in cetane) } \\
\text { at } 25^{\circ} \mathrm{C}\end{array}$ & $\begin{array}{c}\text { Middleman's } \\
\text { Data on Polyiso- } \\
\text { butylene BASF } \\
\text { B-50 at } 23.9^{\circ} \mathrm{C}\end{array}$ \\
\hline 0.0048 & $\ldots \ldots$ & $\ldots \ldots$ & 2.45 \\
.012 & $\ldots \ldots$ & $\ldots \ldots$. & 2.4 \\
.024 & $\ldots \ldots$ & $\ldots \ldots$ & 2.3 \\
1.77 & $2 . .55$ & 2.55 & $\ldots \ldots$. \\
5.56 & 2.55 & 2.55 & $\ldots \ldots$. \\
11.1 & 2.5 & 2.5 & $\ldots \ldots$. \\
22.2 & 2.5 & 2.55 & $\ldots .$. \\
\hline
\end{tabular}

\section{Steady State Shearing Flow}

In the previous section we examined the stress from suddenly applied constant rate of shear. After a certain time, as can be observed, the shearing stress reaches a limiting value independent of time. The viscosity, $\eta(\dot{\gamma})$, which is the ratio of this shearing stress to the rate of shear, is given by

$\eta(\dot{\gamma})=\int_{0}^{\infty} W(\dot{\gamma} \xi, \xi)\left(1+\frac{\partial \ln W(\dot{\gamma} \xi, \xi)}{\partial \ln \gamma}\right) d \xi$
In figure 7 we show the experimental values of viscosity as the open circles. The solid circles are the values calculated from (7.1) and (2.6). In the same figure we show the dynamic viscosity $\eta^{\prime}(\omega)$. We observe that the two curves cannot be superposed by a shift along the horizontal axis.

\section{Stress Relaxation After Steady Shear}

In this history the material is initially sheared at a constant rate of shear $\dot{\gamma}$ until the shearing stress is independent of time. The motion stops at time we call zero, and we observe the subsequent decay of the stress as a function of time. The strain history at time $\tau$ is given by

$$
\begin{array}{ll}
\gamma(\tau)=\dot{\gamma} \tau+\gamma_{0}, & \tau \leqslant 0 \\
\gamma(\tau)=\gamma_{0}, & \tau \geqslant 0 .
\end{array}
$$

From (2.1) and the conditions of (8.1) we obtain ${ }^{9}$

$\sigma(t)=\dot{\gamma} \int_{t}^{\infty} W(\dot{\gamma}(\xi-t), \xi)\left(1+\frac{\partial \ln W}{\partial \ln \gamma}\right) d \xi$

In figure 8 and figure 9 we show the experimental results with the open circles and the calculated results with the solid circles. The experimental points fall slightly higher than the calculated values, as the motion of the upper platen would lead one to expect.

At the limit of zero rate of shear, (8.2) reduces to

$$
\frac{\sigma(t)}{\dot{\gamma}}=\int_{t}^{\infty} G(\xi) d \xi
$$

Differentiating (8.3) with respect to $t$ we get

$$
-\frac{\dot{\sigma}(t)}{\dot{\gamma}}=G(t)
$$

In principle we can obtain $G(t)$ from this limit, but this method is not an accurate one. Since in the previous section we mentioned the errors due to the motion of the upper platen, it is interesting to look at an example where these errors are very pronounced. From (8.3) using an integration by parts we obtain

$$
\frac{1}{\dot{\gamma}} \int_{0}^{\infty} \sigma(t) d t=\int_{0}^{\infty} G(t) t d t .
$$

From eq (4.2) by taking the limit as $\omega$ goes to zero, we get

$$
\lim _{\omega \rightarrow 0} \frac{G^{\prime}(\omega)}{\omega^{2}}=\int_{0}^{\infty} G(t) t d t
$$

${ }^{9} \mathrm{For}$ brevity, when $W$ appears in an integral without its arguments, it is understood that they are the same as for the preceding $W$. 


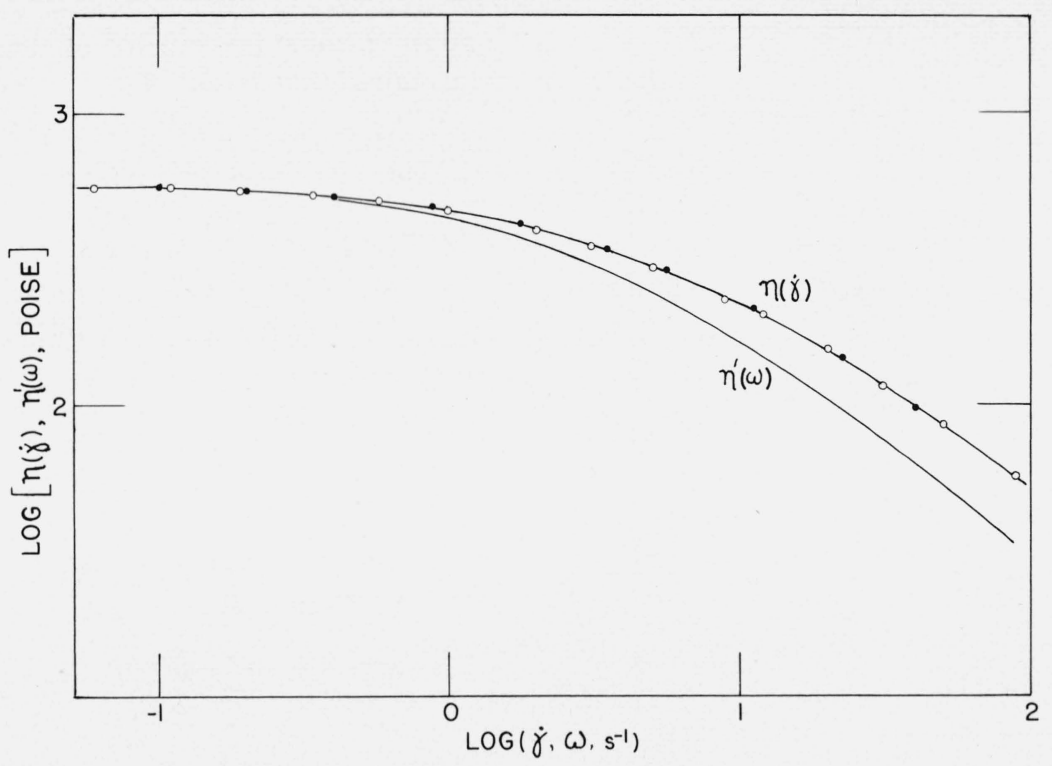

FIGURE 7: The viscosity as a function of rate of shear.

The open circles are experimental data, and the solid circles are calculated values. The dynamic viscosity is also shown for comparison.

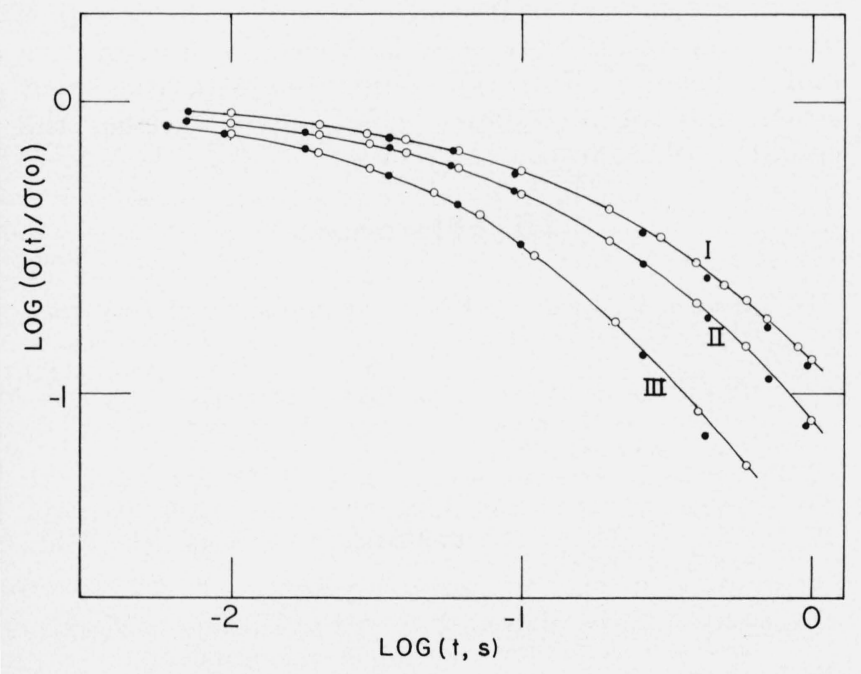

FiguRE 8: Stress relaxation after steady shear, for rates of shear $\dot{\gamma}=0.556 \mathrm{~s}^{-1}(I), \dot{\gamma}=1.77 \mathrm{~s}^{-1}(I I)$, and $\dot{\gamma}=11.1 \mathrm{~s}^{-1}(I I I)$.

The open circles are experimental data, and the solid circles are calculated.

and from (8.5) and (8.6) we obtain

$$
\frac{1}{\dot{\gamma}} \int_{0}^{\infty} \sigma(t) d t=\lim _{\omega \rightarrow 0} \frac{G^{\prime}(\omega)}{\omega^{2}} .
$$

In figure 10 we show a plot of

$$
\dot{\gamma} \int_{0}^{\infty} \sigma(t) d t
$$

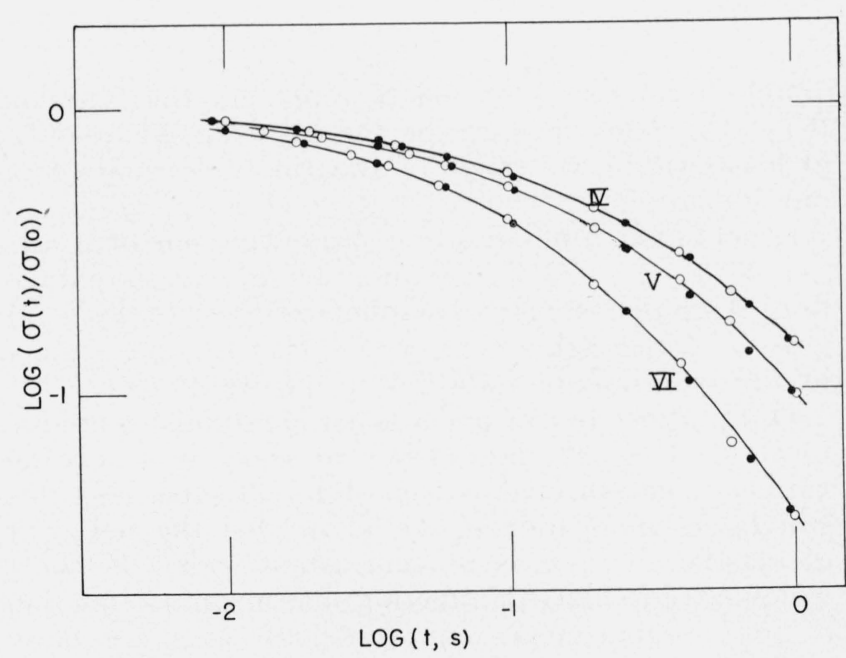

FIGURE 9: Stress relaxation after steady shear, for rates of shear $\dot{\gamma}=0.177 \mathrm{~s}^{-1}(I V), \dot{\gamma}=1.11 \mathrm{~s}^{-1}(V)$, and $\dot{\gamma}=5.56 \mathrm{~s}^{-1}(V I)$.

The open circles are experimental data, and the solid circles are calculated.

versus $\dot{\gamma}$ using different torsion bars. The error introduced by the motion of the platen is very dramatic.

\section{Discussion}

The good agreement between the experimental data and the calculated values shows the validity of the BKZ theory even for materials where the shear relaxation modulus falls very quickly. The relatively 


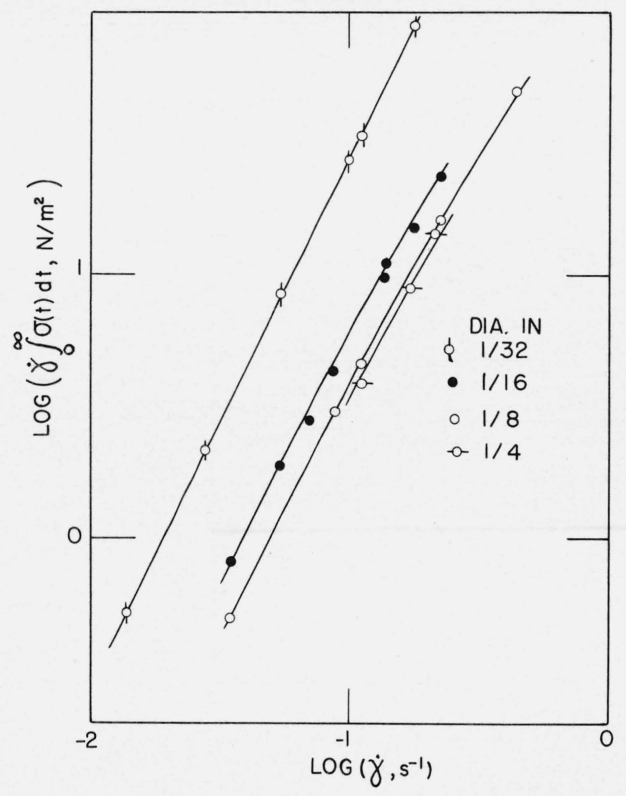

FiguRE 10. Variation of $\dot{\gamma} \int_{0}^{\infty} \sigma(\mathrm{t}) \mathrm{dt}$ as a function of $\dot{\gamma}$ using different torsion bars, where $\sigma(\mathrm{t})$ is the measured stress after the cessation of steady shear.

simple form that we used to represent the function $W(\gamma, t)$ seems to describe the behavior adequately, at least within the range of shearing deformations of our experiments. The separation of $W(\gamma, t)$ into a product of two functions, one of time and one of strain, i.e., $W(\gamma, t)=G(t) W_{R}(\gamma)$, may be an oversimplification. Although a more complicated form of $W(\gamma, t)$ gave us better agreement, we felt that the uncertainty in our experimental data did not justify its use.

Our purpose in this paper is not to establish a unique form of $W(\gamma, t)$, but rather to show how a wide variety of measurements from different shear histories can be related. Indeed, we know that the behavior of plasticized PVC is not consistent with a $W(\gamma, t)$ which is a product of a function of strain and a function of time. Experiments on normal stresses are more critically dependent on the values of $W(\gamma, t)$ at long times and large strains. For instance, the first normal stress difference, $\sigma_{11}(t)-\sigma_{22}(t)$, for a suddenly applied constant rate of shear, given by eq (6.1), can be calculated from [4]

$\sigma_{11}(t)-\sigma_{22}(t)=\dot{\gamma}^{2} \int_{0}^{t} W(\dot{\gamma} \xi, \xi)\left(2+\frac{\partial \ln W}{\partial \ln \gamma}\right) \xi d \xi$

In general, if the function $W$ is such that at high values of $\gamma$ we have

$$
\frac{\partial \ln W}{\partial \ln \gamma}<-2
$$

then the maximum of $\sigma_{11}(t)-\sigma_{22}(t)$ will occur at time $t_{m}$ for which

$$
\frac{\partial \ln W\left(\dot{\gamma} t_{m}, t_{m}\right)}{\partial \ln \gamma}=-2 .
$$

For longer times $\sigma_{11}(t)-\sigma_{22}(t)$ will tend to level to a lower value. This behavior of the first normal stress difference is roughly similar to that of the shear stress. There are clear differences however; a comparison of eq (9.1) with eq (6.2) shows that the overshoot for the shearing stress, if it occurs, must do so at an earlier time than $t_{m}$. It may well be possible, however, to find a form of $W(\gamma, t)$ compatible with the shear stress data of this paper which never satisfies the condition (9.2) for any value of $\gamma$.

\section{References}

[1] Bernstein. B., Kearsley. E. A., and Zapas, L. J., Trans. Soc. Rheol. 7, 391-410 (1963).

[2] Zapas, L. J., and Craft, T., J. Res. Nat. Bur. Stand. (U.S.), 69A (Phys. and Chem.), No. 6, 541-546 (1965).

[3] Broadbent, J. M., Kaye, A., Lodge, A. S., and Vale, D. G., Nature 2 17,55 (1968).

[4] Bernstein, B., Acta. Mechanica, II, No. 4, 329-354 (1966).

[5] Bernstein, B., and Fosdick, R. L., Rheologica Acta 9, 106 (1970).

[6] Ferry, J. D., Viscoelastic Properties of Polymers (John Wiley, New York, 1961).

[7] Marvin, R. S., Phys. Rev. 86, 644 (1952).

[8] Zapas, L. J., and Marvin, R. S., A Correction for a Non-Zero Loading Time in Stress Relaxation (in preparation).

[9] Middleman, S., Trans. Soc. Rheol. 13, 123 (1969).

[10] DeWitt, T. W., Markovitz, H., Padden, F. J., and Zapas, L. J., J. Colloid Sci. 10, 174-188 (1955).

(Paper 75A1-648) 